Information Management and Business Review

Vol. 2, No. 1, pp. 46-47, Jan 2011

Book Review

\title{
From jugaad to systematic innovation: the challenge for India
}

\author{
Author: Rishikesha T. Krishnan \\ Review by: *Shiva Kumar Srinivasan \\ Managerial Communications, IIM Kozhikode, India \\ *shiva@iimk.ac.in
}

What is the difference between jugaad and systematic innovation? Why are Indians good with the former but not with the latter? What, if anything, can be done to create a culture of innovation? What must industry do harness a culture of innovation? These are the questions that Rishikesha Krishnan sets out to answer in this book; it brings together the work that he has done in this area for the last 15 years. Krishnan teaches corporate strategy and entrepreneurship: so it is not surprising that he should turn to the problem of jugaad sooner or later. What is interesting about this book however is the fact that as a theorist of this process, he himself is determined to strike out on the path of systematic innovation rather than merely share a few passing insights with the reader. Another way of situating Krishnan's interest in this topic is to consider the analogue of 'reverse innovation' that Western companies have started to work with. What is it that prevents Indian companies from making the best of reverse innovation? This is where industry comes into the picture; if Western companies are able to spot opportunities aplenty for reverse innovation, then, it is only a matter of time before Indian industries work out the modalities necessary to do so. But to do this in a sustainable manner it is important to foster a culture of innovation by understanding what individual innovators are trying to do without imposing outdated criteria of evaluation on them. Krishnan therefore starts out by identifying those within the IIM and business community who have done a lot to spot and support grassroots innovation like Anil Gupta of Sristi, the work of the National Innovation Foundation, the various initiatives taken up by those interested in the theory and practice of innovation, the development of the relevant set of indicators to measure innovation, and a discussion on the need to design an effective system of incentives in the contexts of business, economic, and public policy. Krishnan is also preoccupied with the enormous contribution that governments can play in facilitating a comprehensive innovation policy for the country as a whole; he therefore summarizes, whenever possible, the findings and recommendations of the various committees that have dwelled on these issues as a prelude to stating his diagnosis and recommendation.

It is important, however, to begin with a caveat on what innovation is and what innovation is not. Innovation is neither creation nor invention per se; an innovation is an application that has commercial value. And, in addition, an innovative application must have both 'novelty and utility'. By novelty, Krishnan does not mean something absolutely new in terms of discovery or invention; the focus is rather on the actual context(s) of application. So, for instance, an idea can be imported or exported across domains in order to generate innovative forms of value addition. This value addition happens not because of what is intrinsic to a given idea, but emerges instead from the context of an innovative set of applications that solve problems unexpectedly, or in ways that are more cost-effective. Here the idea is a constant, but the range of applications is the variable: it could be very many in number depending on the circumstances of an application. If we conflate the process of innovation with terms like creation, discovery, and invention (to which it is semantically related but with which it is not synonymous), we will not understand what is at stake in the process of innovation. The subtitle of this book then is an attempt to understand why Indians who do not lack creativity as individuals are not able to forge the kind of teams, firms, ecosystems, and industrial clusters necessary to make the breakthroughs that used to characterize Western economies until recently. Krishnan's book then is an opportunity for not only Indian companies, but for Western firms as well to determine the socio-economic 'conditions of possibility' for designing the process of both systematic and sustainable innovation. This book is not just a description of the challenges and the process involved in doing so; but, most importantly, an invitation to join in the process. Krishnan's task then is cut out; he has to provide his readers with the data, the information, the ideas, and the theoretical frameworks necessary to think-through the demands involved in making a transition from sporadic instances of jugaad to systematic innovation in India. The main thrust of the argument is that India has not fulfilled its potential, and must be willing to engage in an objective discussion on the underlying causes for this under-performance, and take quick corrective action if it 
must take its place in the comity of developed economies. All this is done in the space of five chapters; the most important part of the study though is the final chapter which sets out in some detail 'what needs to be done' given the specific barriers and constraints in the Indian context. In addition to his own set of recommendations, Krishnan also includes the recommendations made in previous reports by a number of committees in the appendix. This style of writing increases the readability of the book as a whole, but there are still a few more parts which could have been packed into appendices. Most of the chapters can be read separately as well since Krishnan has taken the trouble to explain his terms clearly.

What exactly then are Krishnan's policy recommendations? The main policy objective must be to create a 'critical mass' of innovative firms. This may seem like a rather obvious thing to say, but vested interests, misplaced incentive systems, residual forms of socialism, lack of clarity in terms of policy objectives, the stigma of failure, lack of entrepreneurial finance, etc., have taken their toll on the attempt to set up a system that cherishes the spirit of entrepreneurism, and which has sufficient resilience of spirit to partake of 'creative destruction'. It is therefore important to make it easy to set up and register firms, build the requisite infrastructure, allow a wide range of individuals to attempt careers as entrepreneurs, make it possible to spin-off scientific/technical applications into start-ups, expand government support, allow a range of public-private partnerships, persuade NRIs to invest and build 'industrial parks', encourage the participation of private equity and venture capital, construct a vibrant intellectual property regime, and incentivise the behavioural habits and traits of entrepreneurship throughout the educational system. These however are by no means the complete list of things that must be done, but a representative list with which policy makers can get started. Most well-intentioned readers will agree with Krishnan at least in terms of the broad policy objectives and the list of things that must find their place on the policy agenda. But, like in any IIM decision report, there is a second part that follows a policy recommendation which addresses the 'how' of implementation. Though Krishnan does address many of the how elements, the execution of strategy is always a problem whether it is related to corporate strategy or entrepreneurial strategy or a macroeconomic intervention in terms of a policy recommendation. A better editorial strategy for the last chapter might have been to divide it into separate parts addressing the 'what' and the 'how' clearly, especially since there is a lot of scepticism about the how in the Indian context. I would rate this book quite highly in terms of it ability to address the 'so what' objection. The significance of this book for faculty, students, and policymakers pertains precisely to the challenges involved in creating a mindset that will not only take innovation seriously but help readers to make the transition from sporadic attempts at jugaad to thinking-through the challenges of innovation systematically. A separate chapter or a section on the 'but how' part however would increase the usefulness of the book. I do not say this merely because of my preoccupations as a WAC instructor in the IIM system, but because I am reminded of the difficult question that Atal Bihari Vajpayee posed once after agreeing with the policy recommendations of a McKinsey presentation: 'Yeh sab hoga kaise?' Or, to put it in English: 'How will we get buy-in?'. 